\title{
Nonequilibrium dynamics in type-II superconductors with inhomogeneous vortex pinning
}

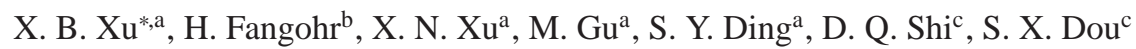 \\ ${ }^{a}$ National Laboratory of Solid State Microstructures, Department of physics, \\ Nanjing University, Nanjing 210093, P. R. China \\ ${ }^{b}$ School of Engineering Sciences, University of Southampton, Southampton SO17 1BJ, United Kingdom \\ ${ }^{c}$ Institute for Superconducting and Electronic Materials, University of Wollongong, NSW 2522, Australia
}

\begin{abstract}
We study numerically the dynamics relating to negative vortex motion in inhomogeneous pinning systems. We show that this dynamical phenomenon results from the internal field effect produced by the growing local barriers with decreasing temperature. We find that the negative motion is characterized by a peak of negative voltage or resistance in resistance-temperature transport measurements. We also demonstrate that the time window to observe the negative motion is determined by the magnitude of driving force in addition to the temperature scanning rate.
\end{abstract}

PACS: 74.25.Fy; 74.25.Qt

Keywords: Langevin dynamics; Negative vortex motion; Inhomogeneous pinning; Relaxation

\section{Introduction}

The off-equilibrium dynamics of superconducting vortex systems has been the focus of significant interest over the last decade[1]. It is well known that, as one of basic interactions with vortices, pinning, including its strength and density, and also its distribution in superconductors, takes the most important role in determining the behavior of vortices in superconductors. Most investigations on off-equilibrium vortex dynamics have been involved with homogeneous pinning systems, which exhibit obvious history, memory, and metastable phenomena in conjunction with strong vortex pinning in magnetic and transport measurements[2]. These off-equilibrium phenomena can be probed, as the typical experimental times become very short compared with the system intrinsic relaxation time scales. Thus, one can study the off-equilibrium dynamics of vortex matter either by adjusting the experimental scanning speed, such as the scanning rates of magnetic field in magnetization-field, or temperature in resistance-temperature, or current in voltage-current measurements, or by adjusting vortex pinning [3-5]. However, for inhomogeneous pinning systems, local strong pinning potentials in such superconductors can trap many vortices, producing an obvious internal field effect: a large local gradient field of vortices occurs inside the superconducting system and hence has dramatic effects on the vortex statics and dynamics [6, 7]. This suggests that the inhomogeneous pinning systems may have completely different dynamical properties as compared with their homogeneous pinning counterparts. However, very few studies, including numerical studies, have focused on the dynamics of inhomogeneous pinning systems so far.

\footnotetext{
*Corresponding author. Tel: 8625 83593076; Fax: 862583595535

Email address: xxb@nju.edu.cn (X. B. Xu)
}

Recently, based on a Langevin molecular dynamics simulation over a short time scale, we found that the interactions between vortices wandering in an inhomogeneous pinning landscape produced unusual dynamics[8]. Driven vortices climbing towards a high barrier will move along the opposite direction to the force as the force is decreasing or withdrawn. An intriguing puzzle naturally arises: in resistance-temperature measurements of superconductors, does vortex matter show negative mobility in the case of growing potential barriers with decreasing temperature and how can the dynamics be probed in experiments? Indeed, as shown here, we extend our previous study of the negative motion with the aim of studying the combined effects of inhomogeneous disorder and thermal fluctuations.

In this work, based on the strong-weak pinning model which is characterized by different regions with stronger and weaker pinning forces (shown in the inset of Fig. 1(a)[8]), we study numerically for the first time the negative motion as a function of temperature in type II superconductors. In fact, the inhomogeneous vortex pinning systems have been widely studied with molecular dynamical simulation, see such as Ref.[9] and Ref.[10]. The driving force is in the $\mathrm{x}$ direction, while the applied current and magnetic field are in $\mathrm{y}$ and $\mathrm{z}$ directions (perpendicular to the paper face) respectively in the infinite plane. The vortices will move along the $\mathrm{x}$ direction as the driving force is large enough. We have found that (1) the enhanced vortex pinning with decreasing temperature produces a growing potential barrier for the moving vortices at a fixed external driving force, resulting in negative vortex motion; (2) the negative motion manifests itself as a peak of negative voltage or resistance in resistance-temperature transport measurements; (3) the time window to observe the negative motion is determined by the magnitude of the driving force in addition to the temperature scanning rate. 


\section{Model and Simulation}

The overdamped Langevin equation of motion for a vortex in position $\mathbf{r}_{i}$ is [11]

$$
\mathbf{F}_{i}=\sum_{j \neq i}^{N_{\mathrm{v}}} \mathbf{F}^{\mathrm{vv}}\left(\mathbf{r}_{i}-\mathbf{r}_{j}\right)+\sum_{k}^{N_{\mathrm{p}}} \mathbf{F}^{\mathrm{vp}}\left(\mathbf{r}_{i}-\mathbf{r}_{k}^{\mathrm{p}}\right)+\mathbf{F}^{\mathrm{L}}+\mathbf{F}_{i}^{\mathrm{T}}=\eta \frac{d \mathbf{r}_{i}}{d t}
$$

where $\mathbf{F}_{i}$ is the total force acting on vortex $\mathrm{i}, \mathbf{F}^{\mathrm{vv}}$ and $\mathbf{F}^{\mathrm{vp}}$ are the forces due to vortex-vortex and vortex-pin interactions, respectively, $\mathbf{F}^{\mathrm{L}}$ is the driving force due to the current $\mathbf{J}\left(\mathbf{F}^{\mathrm{L}} \propto \mathbf{J} \times \hat{\mathbf{z}}\right)$ and $\mathbf{F}^{\mathrm{T}}$ is the thermal stochastic force, $\eta$ is the Bardeen-Stephen friction coefficient: $\eta \propto \phi_{0} B_{\mathrm{c} 2} / \rho_{\mathrm{n}}, N_{\mathrm{v}}$ the number of vortices, $N_{\mathrm{p}}$ the number of pinning centers, and $\mathbf{r}_{k}^{\mathrm{p}}$ the position of the $k$ th pinning center. The vortex-vortex interaction has the form of a logarithmic potential (which is effectively for calculating the interaction energy and force between two pancakes[12, 13]), $U_{v}=2 \varepsilon_{0} \sin (\lambda / \mathbf{r})$, where $\varepsilon_{0}=\phi_{0}^{2} /\left(4 \pi \mu_{0} \lambda^{2}\right)$, s the length of the vortex, $\lambda$ is the London penetration depth, $\phi_{0}$ is the flux quantum, and $\mu_{0}$ the vacuum permeability. The vortex-vortex repulsion force is, $\mathbf{F}^{\mathrm{vv}}\left(\mathbf{r}_{i}-\mathbf{r}_{j}\right)=\left(\phi_{0}^{2} s\right)\left(2 \pi \mu_{0} \lambda^{2}\right)^{-1}\left(\mathbf{r}_{i}-\mathbf{r}_{j}\right)\left(\mid \mathbf{r}_{i}-\right.$ $\left.\mathbf{r}_{j} \mid\right)^{-2}=f_{v v}\left(\mathbf{r}_{i}-\mathbf{r}_{j}\right)\left(\left|\mathbf{r}_{i}-\mathbf{r}_{j}\right|\right)^{-2}$. We employ periodic boundary conditions and cut off the logarithmic vortex-vortex repulsion potential smoothly[14]. The pinning force is attractive: $\mathbf{F}^{\mathrm{vp}}\left(\mathbf{r}_{i}-\mathbf{r}_{k}^{\mathrm{p}}\right)=-f_{\mathrm{pv}}\left(r_{i k} / r_{\mathrm{p}}\right) \exp \left(-\left(r_{i k} / r_{\mathrm{p}}\right)^{2}\right) \hat{\mathbf{r}}_{i k}$, where $f_{\mathrm{pv}}$ tunes the strength of this force and $r_{\mathrm{p}}$ determines its range[15]. We assume $f_{\mathrm{pv}} \propto B_{c 2}^{2}\left(1-B / B_{c 2}\right) \xi^{2} / \kappa^{2}$ as core pinning is considered[16], where $\kappa=\lambda / \xi, B_{\mathrm{c} 2}$ depends on the temperature via upper critical field $B_{\mathrm{c} 2}(T)=B_{\mathrm{c} 2}(0)\left(1-\left(T / T_{c}\right)^{2}\right)$ (The form for $B_{\mathrm{c} 2}(T)$, also for $\lambda(T)$ and $\xi(T)$ in the following context, is correct in the Ginzburg-Landau theory when $T \approx T_{c}$, while providing a good fit to the BCS form over the whole temperature range[17]). The thermal force is implemented with a Box-Müller random number generator and has the properties $\left\langle F_{i}^{\mathrm{T}}\right\rangle=0$ and $\left\langle F_{i}^{\mathrm{T}}(t) F_{j}^{\mathrm{T}}\left(t^{\prime}\right)\right\rangle=2 \eta k_{\mathrm{B}} T \delta_{i j} \delta\left(t-t^{\prime}\right)$ at a given temperature $T$. We use $\lambda(T) / \lambda(0)=\left(1-T / T_{\mathrm{c}}\right)^{-1 / 2}$ and $\xi(T) / \xi(0)=\left(1-T / T_{\mathrm{c}}\right)^{-1 / 2}$ [17]. The average speed of the vortices is $\left\langle V_{\mathrm{x}}\right\rangle=\frac{1}{N_{\mathrm{v}}} \sum_{i}^{N_{\mathrm{v}}} v_{\mathrm{x} i}$ which is proportional to the resulting voltage. We normalize lengths by $\lambda_{0}=\lambda(0)$, forces by $f_{0}=\left(\phi_{0}^{2} s\right)\left(2 \pi \mu_{0} \lambda_{0}^{3}\right)^{-1}$, and time by $\tau_{0}=\lambda_{0} \eta(0) / f_{0}$. All quantities shown in the following figures are expressed in these simulation units.

We perform voltage-temperature simulations with a Lorentz driving force along the $\mathrm{x}$ axis. The total number of vortices $N_{v}=900$ is used. Similar results are obtained for larger systems. We employ $\lambda_{0}=690 \AA, s=12 \AA$, while the pinning strength at zero temperature is $f_{p v 0}=20 f_{0}$, and $\eta_{0}=$ $1.4 \times 10^{-17} \mathrm{~kg} / \mathrm{s}$ and, unless specified otherwise, the temperature scanning rate is $d T / d t=-0.01 T_{c} / t_{0}$, the driving force $F^{L}=0.2 f_{0}, B=0.03 B_{\mathrm{c} 20}$ and $r_{\mathrm{p}}=0.2 \lambda$.

\section{Results and Discussion}

Fig. 1 (a) shows a typical plot of the average velocity of the vortices in the $x$-direction, $V_{\mathrm{x}}$, against the reduced temperature $\mathrm{T} / \mathrm{T}_{\mathrm{c}}$ (open circles) for descending temperature. Negative voltage can be seen in the temperature regime from $\mathrm{T} \approx 0.96 \mathrm{~T}_{\mathrm{c}}$ to
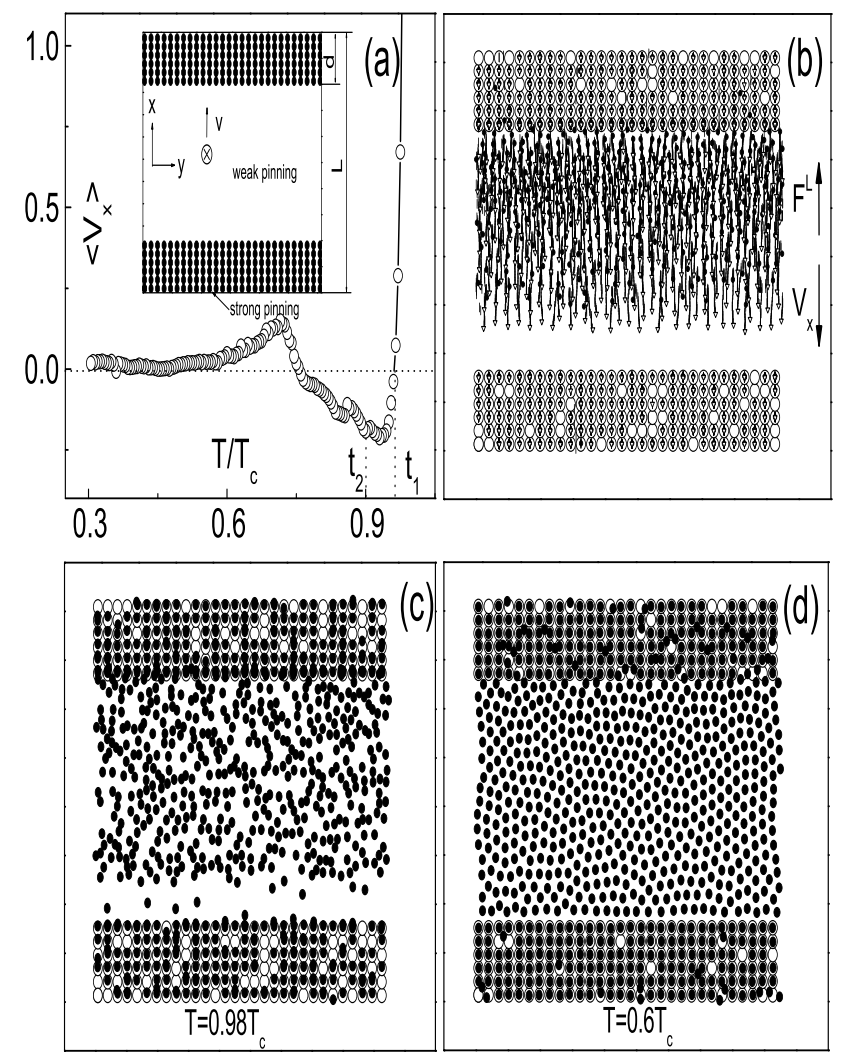

Figure 1: (a) The negative vortex motion in the velocity $\mathrm{V}_{\mathrm{x}}$ (open circles) as a function of temperature. For $0.76 \mathrm{~T}_{c}<\mathrm{T}<0.96 \mathrm{~T}_{c}$, the average voltage of vortices is negative, showing that the motion of vortices is in the opposite direction to the driving force. Inset: Schematic diagram of inhomogeneous pinning model. The black spots and empty squares denote the strong and weak pinning centers, respectively. (b) Vortex motion trajectories (from beginning $\bullet$ to end $\nabla$ ) for $0.9 \mathrm{~T}_{c}<\mathrm{T}<0.96 \mathrm{~T}_{c}$ (corresponding to the temperature region from $\mathrm{t} 1$ to $\mathrm{t} 2$ in (a).) Note that the local pinning (open circles) barrier for the climbing vortices is growing with decreasing temperature. As the repulsive force exerted on the moving vortices by the trapped vortices becomes larger than the driving force, the vortices in the weak pinning region move in the opposite direction to the driving force. The arrows up and down show the directions of driving force and vortex motion, respectively. (c) Vortex (filled circles) distribution at $\mathrm{T}=0.98 \mathrm{~T}_{c}$, showing that the vortices are disordered but comparatively homogenous. In other words, the internal field effect which causes the negative vortex motion is not important. (d) $\mathrm{T}=0.6 \mathrm{~T}_{c}$, where the vortex-vortex interactions are important, so more and more vortices are elastically depinned. This leads to a homogenous vortex distribution and thereby a weak internal field effect, which is similar to that at high temperature. We have used $B=0.06 B_{\mathrm{c} 20}$ and $r_{\mathrm{p}}=0.05 \lambda$. 


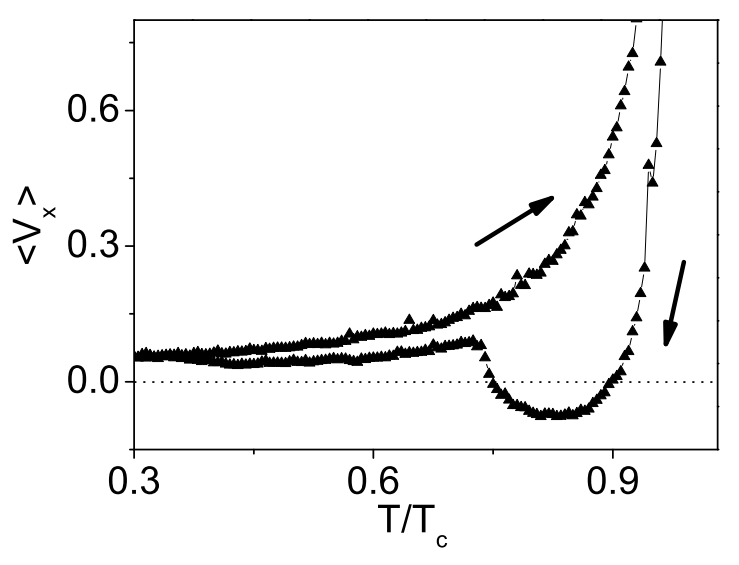

Figure 2: A typical clockwise $\mathrm{V}_{x}-\mathrm{T} / \mathrm{T}_{c}$ loop in a cooling/warming cycle. The arrows indicate the evolution of temperature. It is clear that the negative voltages occur only for decreasing temperature, while the voltage of vortices is always positive for increasing temperature. This suggests that the equilibrium vortex states for the inhomogeneous pinning systems can easily be probed in the warming process. We have used $r_{\mathrm{p}}=0.05 \lambda$.

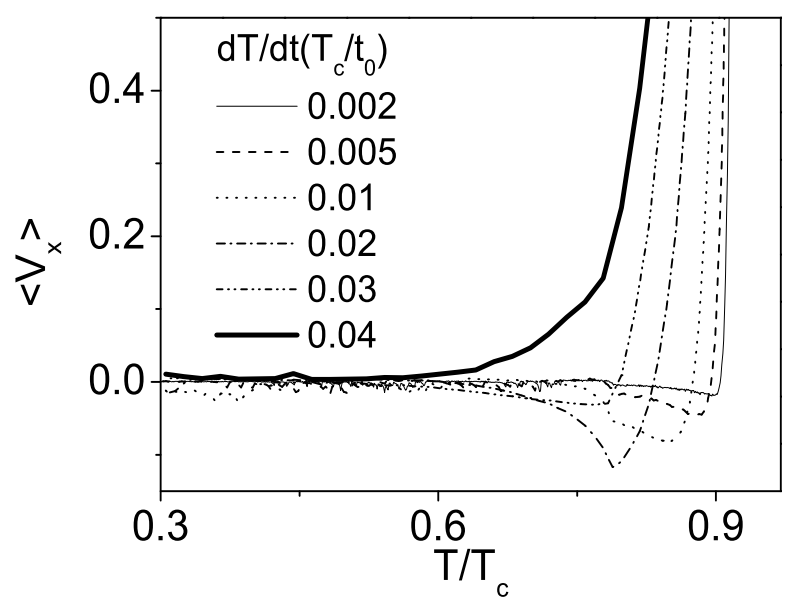

Figure 3: Effect of the temperature scan rate $\mathrm{d} T / \mathrm{d} t$ on the negative motion for $F^{L}=f_{0}$. With increasing $\mathrm{d} T / \mathrm{d} t$, two features of the dynamics can be seen: (1) the peaks of negative vortex voltage shift towards low temperature; (2) the magnitude of the negative voltage peak first becomes larger and reaches its maximum for $\mathrm{d} T / \mathrm{d} t=0.02 \mathrm{~T}_{c} / \mathrm{t}_{0}$, and then become smaller. This means that the temperature scanning rate has to be chosen properly in order to observe the negative motion.

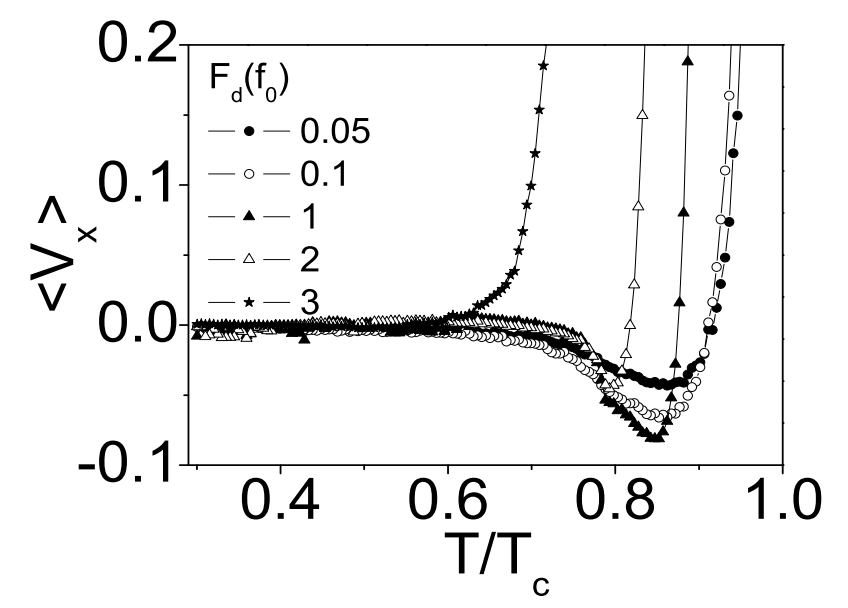

Figure 4: Effect of the driving force on the negative motion. As the driving force increases, two features of the dynamics are clearly seen (similar to those results caused by changing $\mathrm{d} T / \mathrm{d} t$, as shown in Fig. 3): (1) the peaks of negative vortex voltage shift towards low temperature; (2) the magnitude of the negative voltage peak first becomes larger and reaches its maximum for $\mathrm{d} T / \mathrm{d} t=0.02 \mathrm{~T}_{c} / \mathrm{t}_{0}$, and then become smaller. The simulation results indicate that the driving force changes the system intrinsic relaxation time scales and thus the time window to observe the negative motion.

$\mathrm{T} \approx 0.76 \mathrm{~T}_{\mathrm{c}}$. The simulated results show three distinct regimes as follows.

At higher temperatures $\left(\mathrm{T}>0.96 \mathrm{~T}_{\mathrm{c}}\right)$, both vortex-pinning interactions and vortex-vortex interactions are weak, and thermal fluctuations are strong. So the vortices are disordered but comparatively homogenously distributed in the simulation cell, as shown in Fig. 1(c). The vortices move along the direction of the driving force. That is, the average velocity of the vortices is positive. With decreasing temperature, the pinning force becomes larger and thereby more vortices are trapped by the local pinning centers. The vortex-vortex repulsive interactions also become larger with decreasing temperature, resulting in a growing local barrier for the moving vortices driven by the driving force. Thus, the average velocity of vortices becomes smaller and equals zero for $\mathrm{T} \approx 0.96 \mathrm{~T}_{c}$

In the negative motion region $\left(0.76 \mathrm{~T}_{\mathrm{c}}<\mathrm{T}<0.96 \mathrm{~T}_{\mathrm{c}}\right)$, the motion of vortices is in the opposite direction to the driving force. With further decreasing temperature, the repulsive force exerted on the moving vortices in the weak pinning region by the trapped vortices in the strong pinning region becomes dominant over the driving force. The vortices in the weak pinning region go back to their equilibrium positions, which is characterized by negative voltage or resistance [8, 18]. At $\mathrm{T}=0.93 \mathrm{~T}_{c}$, the negative velocity reaches its maximum (see the inset of Fig. 1 a)). As the moving vortices approach their equilibrium positions, the repulsive force exerted on these vortices become smaller, and thus the negative velocity is reduced.

At low temperatures $\left(\mathrm{T}<0.76 \mathrm{~T}_{\mathrm{c}}\right)$, the vortex-vortex interactions become more important, and thus more and more vortices are elastically depinned. This leads to a homogeneous vortex distribution, as shown in Fig. 1(d). The local energy barrier and the resulting internal field effect are weakening. As a result, the vortices move along the direction of the driving force, i.e., 
the average voltage becomes positive, as shown in the inset of Fig. 1(a). Besides, it can be seen that the vortices in the weak pinning region become ordered, because the elastic interactions between vortices are dominant in such low temperatures. This is similar to the vortex states confined in nanoscopic and mesoscopic superconductors [19].

Fig. 2 demonstrates the hysteretic behavior of vortices due to the inhomogeneous pinning. It can be seen that the average voltage of vortices is always positive for increasing temperature, in contrast to those for decreasing temperature. At low temperatures the internal field effect is weak due to comparatively homogeneous vortex distributions, so the vortices can easily stay in their equilibrium positions. Thus, when the vortex system enters the negative motion regime, although local energy barriers exit due to local strong pinning centers, the moving vortex can only feel the driving force. So the direction of vortex motion is the same as that of the driving force in the warming process. Apparently, this type of clockwise $\mathrm{V}_{x^{-}}$ $\mathrm{T} / \mathrm{T}_{\mathrm{c}}$ loop in one cooling/warming cycle is the fingerprint of the negative motion induced by inhomogeneous pinning.

As one sort of off-equilibrium dynamical phenomenon, the time window to observe the negative motion is determined by the competition between the speed of vortex diffusion and an temperature scanning rate. Fig. 3 shows the $\mathrm{V}_{x}-\mathrm{T} / \mathrm{T}_{\mathrm{c}}$ curves for several temperature ramping rates $\mathrm{d} T / \mathrm{d} t$ at fixed pinning and driving forces. It is apparent that the increasing temperature scanning rate results in the two following effects. First, the peaks of negative voltage shift towards lower temperature. It is expected that, for different $\mathrm{d} T / \mathrm{d} t$, it will take almost the same time for the vortices moving from the positions where they start to move negatively to their equilibrium positions. This means that, for larger $\mathrm{d} T / \mathrm{d} t$, it is at lower temperature that one can observe the equilibrium state characterized by a negative voltage peak. Second, the magnitude of the negative voltage peak becomes larger at first and reach its maximum for $\mathrm{d} T / \mathrm{d} t=0.02 \mathrm{~T}_{c} / \mathrm{t}_{0}$, as shown in Fig. 3, and then become smaller. For small $\mathrm{d} T / \mathrm{d} t$, the negative voltage peak is observed in the high temperature region. The vortex pinning is weak, and thermal fluctuations are strong for high temperature, so the internal field effect induced by inhomogeneous pinning is weak. Thus, the resulting negative motion of vortices is not marked. Moreover, for large $\mathrm{d} T / \mathrm{d} t$, the negative motion is probed at low temperature, where the enhanced vortex-vortex interactions weaken the internal field effect. Hence the corresponding negative motion is not obvious, similar to that at high temperatures. The simulation results suggest that the temperature scanning rate has to be chosen properly in order to observe the negative motion.

Finally, we concentrate on the effect of driving force on the negative motion. In Fig. 4 we show the $\mathrm{V}_{\mathrm{x}}-\mathrm{T} / \mathrm{T}_{\mathrm{c}}$ curves for different values of driving force at fixed $\mathrm{d} T / \mathrm{d} t$. As we can see, driving force strongly changes the dynamics and also the time window for probing the negative motion. At low driving force, most of the moving vortices are not far away from their equilibrium positions. Thus, the resulting internal gradient field is small, and the negative motion is thus not obvious. At high driving force, the effective negative force is small due to the offset- ting influence of the high driving force. This results in a small negative voltage. Furthermore, with increasing driving force, a small diffusion force results in a long negative diffusion time for the moving vortices to reach their equilibrium positions. Therefore, the time window to observe the negative motion moves to low temperature for high driving force.

\section{Summary}

In conclusion, we have numerically studied the dynamics relating to negative vortex motion, which is induced by the growing local barriers, which are dependent on temperature. We have found that the negative motion results from the combined effects of the diffusion of negative vortex motion and the elastic depinning with decreasing temperature. Moreover, the simulation results reveal that both the temperature scanning rate and the magnitude of the driving force determine the time window to observe the negative motion. These numerical results can be tested experimentally by looking for negative resistance peaks in the temperature-resistance curves.

\section{Acknowledgments}

This work is supported by the National Basic Research Programme of China under 2006CB705600, the National Natural Science Foundation of China under 10674060, and the Natural Science Foundation of Jiangsu Province under BK2006109.

\section{References}

[1] S. Bustingorry, L. F. Cugliandolo, and D. Domínguez, Phys. Rev. B 75 (2007) 024506.

[2] M. Nicodemi, and H. J. Jensen, J. Phys. A: Mat. Gen. 34 (2001) 8425.

[3] Y. Yeshurun, A. P. Malozemoff, A. Shaulov, Rev. Mod. Phys. 68 (1996) 911.

[4] Z. L. Xiao, E. Y. Andrei, and M. J. Higgins, Phys. Rev. Lett. 83 (1999) 1664.

[5] X. B. Xu, L. Zhang, S. Y. Ding, X. Leng, Z. H. Wang, and X. L. Wang, Supercond. Sci. Technol. 18 (2005) 758.

[6] E. H. Brandt, Rep. Prog. Phys. 59 (1995) 1465.

[7] E. H. Brandt and M. Indenbom, Phys. Rev. B 48 (1993) 12893.

[8] X. B. Xu, H. Fangohr, S. Y. Ding, M. Gu, T. B. Tang, Z. H. Han, D. Q. Shi, and S. X. Dou, Phys. Rev. B 75 (2007) 224507.

[9] J. F. Wambaugh, F. Machesoni, and F. Nori, Phys. Rev. B 67 (2003) 144515.

[10] C. Reichhardt, C. J. Olson, and F. Nori, Phys. Rev. B 61 (2000) 3665.

[11] C. J. Olson, C. Reichhardt, R. T. Scalettar, G. T. Zimányi, and Niels Grønbech-Jensen, Phys. Rev. B 67 (2003) 184523.

[12] J. R. Clem, Phys. Rev. B 43 (1991) 7837.

[13] C. Reichhardt and C. J. Olson, Phys. Rev. B 65 (2002) 100501(R).

[14] H. Fangohr, A. Price, S. Cox, P. A. J. de Groot, G. J. Daniell, and K. S. Thomas, J. Comput. Phys. 162 (2000) 372.

[15] B. Y. Zhu, F. Marchesoni, and Franco Nori, Phys. Rev. Lett. 92 (2004) 180602.

[16] H. J. Jensen, A. Brass, A. C. Shi, and A. J. Berlinsky, Phys. Rev. B 41 (1990) 6394.

[17] M. Tinkham, Introduction to Superconductivity, McGraw Hill, New York (1975).

[18] L. F. Goodrich, J. Moreland, and A. Roshko, IEEE Trans. On Appl. Supercond. 27 (1991) 1194.

[19] H. J. Zhao, V. R. Misko, F. M. Peeters, V. Oboznov, S. V. Dubonos, and I. V. Grigorieva, Phys. Rev. B 78 (2008) 104517. 